\title{
Étude d'un appareil permettant la reproduction de l'onde marée dans un modèle d'estuaire
}

\section{Study of an apparatus for the reproduction of tides in a model estuary}

\author{
PAR J. VALEMBOIS \\ DOCTEUR Ès SGIENCES \\ Ingénieur au Laboratome Natronal d'Hydrauligue (Chatou)
}

English synopsis p. 635

\section{INTRODUCTION}

En vue de la construction, au Laboratoire National d'Hydraulique, du modèle d'un estuaire important, nous avons été amenés à étudier, pour la réalisation de l'onde marée dans ce modèle, un appareil régulateur de niveau que nous décrivons ci-après. Le problème est relativement simple, car l'estuaire est reproduit jusqu'à la limite amont de sa partie maritime, et la limite aval du modèle est telle que la loi de marée y est la même sur toute sa largeur.

Nous exposerons d'abord les raisons qui nous ont conduits à choisir, parmi les appareils utilisés jusqu'à présent pour réaliser les marées sur modèle, tel type plutôt que tel autre. Après avoir étudié théoriquement les caractéristiques optima à donner aux divers éléments de ce dispositif, nous indiquerons la façon dont nous comptons le réaliser pratiquement. On verra que nous arrivons à un système très simple, d'un réglage facile et dont les éléments pourraient servir à résoudre des problèmes plus complexes.

Chorx de L'Appareil rígulateur de nIVEaU

Sur un modèle d'estuaire comme celui que nous considérons, il faut reproduire la marée uniquement dans sa section aval. Une fois la rugosité des fonds bien réglée tout au long du modèle, la similitude de la propagation de l'onde marée sera réalisée, si, bien entendu, les échelles sont convenablement choisies.

Nous serons amenés à modifier les formes de l'estuaire dans sa partie amont. Les volumes d'eau introduits à chaque marée dans l'estuaire seront donc différents selon les essais, pour un même coefficient de marée. La limite aval du modèle a été choisie suffisamment près de la mer pour que la courbe de marée n'y soit pas influencée par ces modifications. Ceci nous a conduits à éliminer, dès le début, des dispositifs comme celui qui consiste en un plongeur introduit suivant une loi bien déterminée dans l'eau du modèle à sa limite aval, la loi du mouvement vertical de ce plongeur étant réglée par approximations successives en même temps que la rugosité du modèle jusqu'à ce que l'on obtienne dans tout le modèle une loi de marée conforme à la loi réelle. C'est qu'en effet cet appareil régit le volume d'eau introduit dans le modèle, et non le niveau de l'eau dans la section aval. On doit régler à nouveau la loi du mouvement vertical du plongeur chaque fois que l'on fait dans le modèle un changement de formes modifiant le volume d'eau introduit au cours d'une marée. Un appareil à plongeur prendrait 
d'ailleurs une taille prohibitive pour un modèle de l'importance de celui que nous étudions (1).

Le procédé suivant permettrait, s'il était réalisable pratiquement pour de grands modèles, de reproduire très simplement une marée dans n’importe quelles conditions. Il consiste à introduire dans la partie amont du modèle un débit constant (supérieur au débit maximum de flot), et à en évacuer une partie au moyen d'un déversoir dont l'arête se déplace verticalement suivant la loi de marée à réaliser, cette arête étant suffisamment longue pour que les variations du débit évacué n'entraînent qu'une variation de charge inférieure à l'erreur admise. En pratique, ceci nous aurait conduits à une longueur d'arête prohibitive, de l'ordre de plusieurs centaines de mètres.

La longueur de l'arête pourrait être diminuée en réglant a priori le débit introduit dans le modèle en fonction du temps pour que le débit déversé se maintienne à peu près constant. Comme cela ne peut se faire que par approximations successives, le procédé serait assez long à mettre en ceuvre. Ces raisons ont amené les expérimentateurs, dans beaucoup de cas, à prévoir un dispositif de régulation. Par exemple, on peut, dans l'appareil précédent, régler à chaque instant le débit introduit dans le modèle en fonction de l'écart existant à cet instant entre le niveau théorique et le niveau récl de l'eau dans la section aval. Ce procédé n'est cependant pas parfait, car on est amené à prévoir un dispositif tranquillisateur sérieux pour l'introduction de l'eau, el le réglage du débit introduit ne se fait sentir dans le modèle qu'arec un retard important dû̀ à ce dispositif, ce qui peut gêner sensiblement la régulation.

Cette difficulté conduit à règler non pas sur le débit introduit dans le modèle, mais sur le débit évacué. Certains expérimentateurs ont utilisé, par exemple, une arête déversante qui était actionnéc par le dispositif régulateur, de facon à faire remonter le niveau s'il était trop bas dans le modèle, et inversement. Le calcul montro que si l'on veut obtenir un réglage suffisamment rapide, il faut prévoir une longueur de déversoir importante, donc un appareil de régulation de grande puissance. D'autre part, comme d'un moment à l'autre de la marée le débit évacué varie dans de larges proportions, la charge sur le déversoir prend au cours de la marée des valeurs très différentes. Or, la loi donnant le débit en fonction du niveau n'est pas linéaire. L'efficacité du réglage par le déversoir, pour une

(1) Le modèle dont nous parlons iei est un modèle où l'estuaire a une importance prépondérante devant la pleine mer. Naturellement, dans le cas contraire, l'emploi d'un dispositif à plongeur se justifierait pour des modèles suffisamment petits. même vitesse de montée ou de descente de l'arête, n'a donc pas la même valeur aux divers instants de la marée.

Le procédé qui consiste à évacuer le débit excédentaire par une vanne de fond élimine tous ces inconvénients. Si cette vanne est suffisamment basse par rapport au niveau de l'eau. les variations de niveau dues à la marée n'introduisent pas une variation relative de charge trop importante, et un orifice de dimensions relativement réduites, manœuvré par un dispositif régulateur de faible puissance, suffit à évacuer de gros débits. C'est pourquoi nous avons adopté ce dispositif, que nous allons étudier dans la suite de cet exposé, en vue de son application aux modèles d'estuaire.

\section{ETUde de L'APpareil REGULATEUR DE NIVEAU}

Comme nous l'avons indiqué plus haut, cet appareil est destiné à reproduire la variation du niveau de l'eau avec la marée dans la section aval d'un modèle d'estuaire, de telle façon que le réglage soit indépendant des volumes d'eau introduits dans le modèle par la marée.

\section{II comporte :}

- Une alimentation en eau fournissant un débit constant $Q$ supérieur au débit maximum de flot. Une tranquillisation efficace la précède. Ce débit est de l'ordre de $200 \mathrm{l} / \mathrm{s}$ pour le modèle que nous considérons.

- Une vanne de fond à orifice réglable, qui puisse évacuer un débit égal à la somme de $Q$ et du débit maximum de jusant, soit ici $400 \mathrm{l} / \mathrm{s}$ environ.

- Un système de réglage qui permette la commande de la vanne de réglage en fonction de l'écart existant à chaque instant entre le niveau dans la section aval du modèle et la valeur théorique de ce niveau.

\section{Notatrons (voir la figure 1 )}

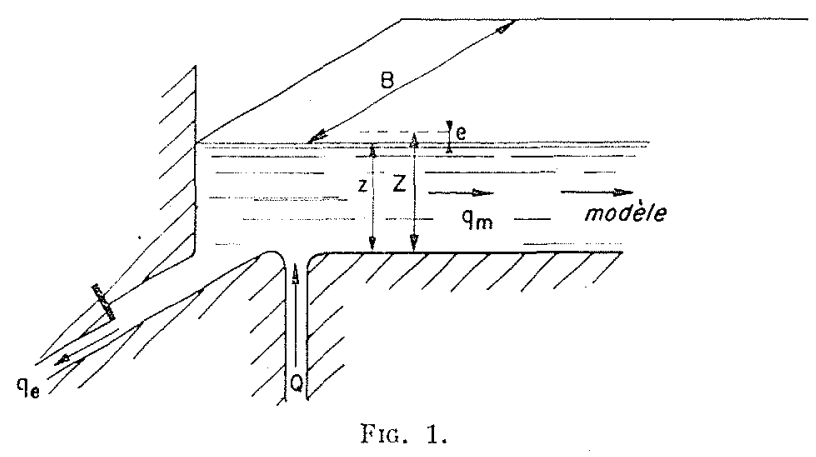


$Z(t)$ : Loi de niveau à réaliser dans la section aval. Nous l'appellerons : niveau théorique.

$z(t)$ : Niveau existant à I'instant $t$ dans la section aval. Ce sera le niveaur réel.

$e(t)=z-Z:$ Ecart entre le nivean réel et le niveau théorique à l'instant $t$. Nous l'appellerons écart réel.

$Q$ : Débit constant introduit dans le modèle.

$q_{\mathrm{e}}(t)$ : Débit évacué par la vanne de fond.

$q_{\mathrm{m}}(\boldsymbol{t})$ : Valeur du débit de marée dans la section aval de l'estuaire, $q_{\mathrm{m}}$ sera positif pour le courant de flot, négatif pour le jusant.

$a$ : Valeur de la vitesse de propagation des ondes de gravité dans la section aval.

B : Largeur de la section aval (1).

\section{Calcul des Caractéristigues du RÉGulateur}

Nous appellerons compartiment à marée l'extrémité du modèle située après la section aval. Il comporte l'alimentation en eau et la vanne d'évacuation. Nous verrons que ce compartiment doit être autant que possible court et profond. L'écart $e$ est mesuré dans la section aval.

Si l'on néglige le débit correspondant aux variations de niveau dans le compartiment à marée, on peut écrire :

$$
\mathrm{Q}=q_{e}+q_{m}
$$

Comme $\mathrm{Q}$ est constant :

$$
\frac{d q_{e}}{d t}+\frac{d \eta_{m}}{d t}=0
$$

$d q_{\mathrm{c}} / d t$ doit être une fonction de l'écart de réglage $e$. L'expérience des spécialistes de la régulation indique qu'il est intéressant d'avoir entre eux une relation de la forme :

$$
\frac{d q_{e}}{d t}=\mathrm{K}\left(e+0_{1} \frac{d e}{d t}\right)
$$

(1) $a$ et $B$ sont des fonctions de $z$. De plus, $a$ n'a pas la même valeur le long de toute la section aval. Nous verrons plus loin que I'on peut, pour obtenir la condition de stabilité de la régulation, prendre les valeurs minimum de $a$ et $B$.
En effet, la proportionnalité de la vitesse de réglage à l'écart assure une correction d'autant plus rapide que celui-ci est plus grand, et l'introduction d'une partie de la dérivée de l'écart permet de réaliser une régulation stable. Enfin, ce cas se traite facilement par le calcul dans sa généralité, ce que l'on ne peut pas faire pour un réglage par tout ou rien.

La difficulté pratique consiste à transformer l'écart $e$ de façon à obtenir un écart corrigé $e_{c}=e+\theta_{1} d e / d t$, è à commander la vanne de réglage de telle façon que la vitesse de variation du débit y soit proportionnelle à cet écart corrigé. Nous décrirons plus loin des dispositifs simples qui réalisent ces deux conditions à peu de frais.

Nous continuerons maintenant le calcul comme si le modèle n'était pas réfléchissant. Cela n'est pas le cas pour un estuaire, mais on doit quand même de cette façon approcher de la réalité, car l'onde réfléchie y est étalée dans le temps (1). La condition que nous allons trouver donnera un ordre de grandeur des caractéristiques de l'appareillage, qu'on modifiera expérimentalement de façon à obtenir une régulation stable.

$\mathrm{Si}$ le modèle n'était pas réfléchissant, nous aurions, entre les variations $d q_{\mathrm{m}}$ du débit $q_{\mathrm{m}}$ et $d z$ du niveau dans la section aval, la relation suivante :

$$
d q_{m}=a \mathrm{~B} d z=a \mathrm{~B}(d Z+d e)
$$

En portant dans (2) les valeurs de $d q_{0}$ et $d q_{\mathrm{m}}$ données par (3) et (4), on obtient:

$$
e+\left(0_{1}+\frac{a \mathrm{~B}}{\mathrm{~K}}\right) \frac{d e}{d t}=-\frac{a \mathrm{~B}}{\mathrm{~K}} \frac{d \mathrm{Z}}{d t}
$$

La formule (5) montre que le réglage pourrait être stable, même sans l'introduction de la dérivée de l'écart.

Cependant, d'autres retards peuvent intervenir pour modifier l'équation (5). Voyons quels sont les principaux.

Tout d'abord, pour mesurer l'écart de réglage $e=z-Z$, on sera obligé d'opérer par l'intermédiaire d'un appareil qui amortisse les variations trop rapides du niveau $z$ (par exemple les rides capillaires), mais qui introduit un léger retard. L'expérience montre que l'on peut arriver à réduire ce retard à une valeur négligeable. D'autre part, le système de commande de la vanne a un certain retard $\tau_{2}$ qui, dans les

(1) Cette approximation est probablement justifiec pour les petites oscillations de réglage. 
dispositifs que nous décrivons plus loin, est de l'ordre de 1/4 de seconde. Enfin, entre le moment où la vamne de réglage est actionnée et le moment où son effet se fait sentir à l'endroit où l'écart de réglage est mesuré, il s'écoule encore le temps $\tau_{3}$ de propagation de l'onde depuis la vanne à cette section. C'est pour réduire ce retard que, comme nous l'avons indiqué plus haut, le compartiment à marée devra être étroit et profond. Admettons que $\tau_{3}$ soit aussi de l'ordre de $1 / 4$ de seconde.

On voit que le $d q_{\mathrm{n}} / d t$ que l'on doit faire intervenir dans l'équation (2) n'est plus une fonction de $e(t)$, mais bien de $e\left(t-\tau_{1}-\tau_{2}-\tau_{3}\right)$ et que, si on laisse de côté les termes en $d^{2} e / d t^{2}$, $0_{1}$ doit être remplacé dans (3) par :

$$
\theta_{1}-\left(\tau_{1}+\tau_{2}+\tau_{3}\right)
$$

D'autres retards peuvent intervenir, mais nous avons examiné les plus importants. En pratique, il faut compter, si les précautions que nous avons indiquées sont bien prises, sur un retard de l'ordre de la seconde.

On peut compenser ces retards, soit en augmentant la valeur de $a \mathrm{~B} / \mathrm{K}$, c'est-à-dire pratiquement en diminuant $K$, qui est la vitesse de réglage, soit en augmentant $\theta_{1}$.

Notons aussi qu'il apparait dans l'équation (5) des termes en $d^{2} e / d t^{2}$, avec un coefficient négatif qu'il scrait intéressant de pouvoir annuler (ou rendre positif de façon que l'équation (5) devienne celle d'un système oscillant à l'amortissement critique). Ceci nécessiterait l'introduction dans l'écart corrigé d'une fraction de la dérivée seconde de l'écart réel.

Nous allons maintenant décrire un appareillage qui remplit les conditions ci-dessus.

\section{DESCRIPTION DE L'APPAREIL RÉGULATEUR}

Dans le modèle que nous considérons, la section aval aura $11 \mathrm{~m}$ de largeur et $11 \mathrm{~cm}$ de profondeur maximum.

\section{Vanne de réglage :}

Elle évacue, sous une charge de $1 \mathrm{~m}$, un débit maximum de l'ordre de $400 \mathrm{l} / \mathrm{s}$, par un orifice de $0,50 \mathrm{~m}$ de largeur et de $0,20 \mathrm{~m}$ de hauteur. Afin de pouvoir être commandée par un moteur de faible puissance, elle est du type secteur, sans étanchéité, et équilibrée en poids. Pour un écart corrigé de $1 \mathrm{~mm}$, elle serait actionnée à la vitesse de $1 \mathrm{~cm} / \mathrm{s}$ correspondant à une variation du débit évacué de $20 \mathrm{l} / \mathrm{s}$ en une seconde ( $a \mathrm{~B} / \mathrm{K}=0,6$ seconde). L'effort de commande est de l'ordre de quelques centaines de grammes.
Commande de la vanne de réglage (fig. 2) :

Il s'agit de faire tourner le moteur de commande de la vanne avec une vitesse proportionnelle à l'écart corrigé. Pour cela, on transforme cet écart en une tension continue qui lui est proportionnelle. Cette tension est appliquée à l'entrée d'un amplificateur symétrique. Le moteur de commande de la vanne est un moteur à courant continu à excitation séparée et à deux bobinages d'induit. Un de ces bobinages est branché en dérivation sur la sortie de l'amplificateur. Il est traversé par un courant proportionnel à la tension d'entrée, donc à l'écart corrigé. Pour rendre la vitesse du moteur bien proportionnelle à cet écart, on ajoute à la tension d'entrée une tension proportionnelle à la vitesse du moteur, recueillie sur le deuxième enroulement d'induit qui fonctionne en dynamo tachymétrique (tension de contre-réaction).

On obtient ainsi, avec un retard minime dû simplement à l'inertie du rotor du moteur, une

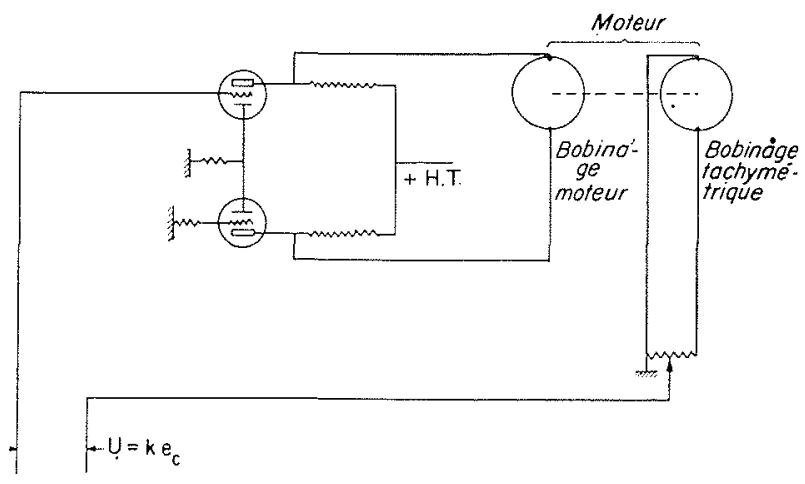

FIG. 2.

vitesse de la vanne de réglage proportionnelle à l'écart corrigé. Le moteur développe une puissance de l'ordre de quelques watts, largement suffisante pour actionner la vanne à la vitesse maximum de $1 \mathrm{~cm} / \mathrm{s}(1$ watt $\sim 10 \mathrm{~kg} \times \mathrm{cm} / \mathrm{s})$.

\section{Détecteur d'écart :}

Ce dispositif a pour objet de transformer l'écart $e=z-Z$ ou l'écart corrigé $e_{c}$ en une tension continue qui lui soit proportionnelle.

Nous ayons étudié plusieurs systèmes, dont l'un est décrit dans une communication faite au congrès de septembre 1949 de l'A.I.R.H. Un autre dispositif, plus simple, est actuellement à l'étude et paraît donner de bons résultats.

\section{Correcteur d'écart:}

Nous pensons, s'il se révèle nécessaire pour certains cas d'introduire la dérivée de l'écart, 
essayer deux méthodes, l'une électrique, l'autre hydraulique, pour transformer l'écart $e$ en :

$$
\mathrm{e}+\theta_{1} d e / d t
$$

\section{Procédé électrique:}

Le circuit ci-après, que nous donnons à titre d'exemple, permet, à partir d'une tension $u$, d'obtenir une tension $u_{c}=\mathrm{K}\left(u-j-\theta_{1} d u / d t\right)$. Si $u$ est proportionnel à l'écart réel, $u_{\mathrm{c}}$ sera proportionnel à l'écart corrigé (fig. 3 ).

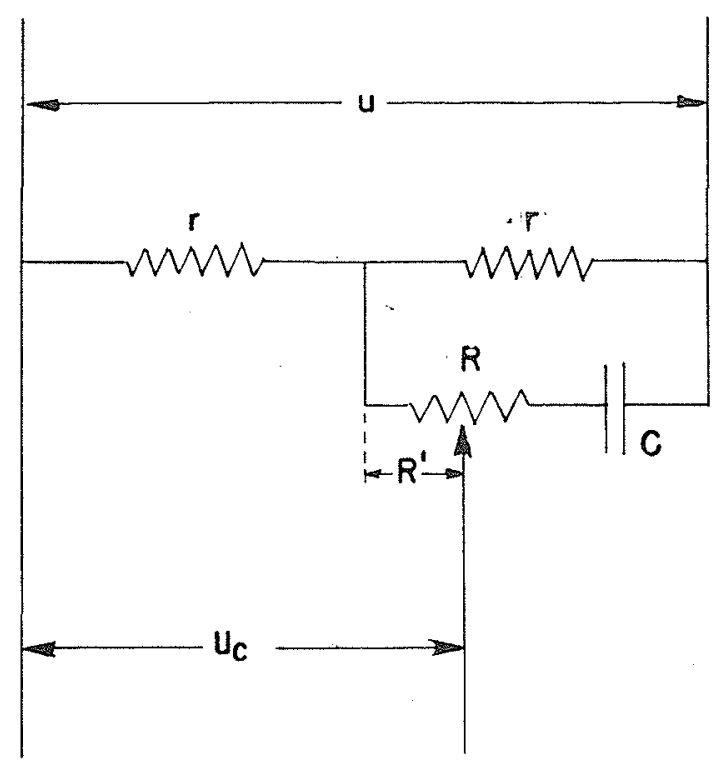

FIG. 3.

$r$ est petit par rapport à $\mathrm{R}$.

$$
\begin{aligned}
& \mathrm{K}=1 / 2 \\
& 0_{1}=\mathrm{CR}^{\prime}
\end{aligned}
$$

L'avantage de ce procédé sur le procédé hydraulique est la facilité du réglage de $\theta_{1}$, qui s'effectue au moyen d'un simple potentiomètre. Il est facile d'obtenir des temps $\theta_{1}$ de l'ordre de plusieurs secondes.

\section{Procédé hydraulique :}

Il consiste à mesurer $z$ par l'intermédiaire d'un pot de mesure calculé de telle façon qu'à sa partie intérieure le niveau soit $z_{0}$ tel que :

$$
z_{c-\mathrm{Z}}=e_{c}=e+\mathrm{R} d e / d t+\mathrm{L} d^{2} e / d t^{2}
$$

Ceci est réalisé par le dispositif de la figure 4.

Le pot de mesure, de section $\mathrm{S}$, est réuni au modèle par un tube fin $\mathrm{T}$, de section $s$ et de longueur $l$. Un piston $\mathrm{P}$ (on utilisera évidem- ment un dispositif ayant le même effet sans qu'il y ait de frottements), fait varier le volume de l'eau comprise dans le pot. Sa section est K S

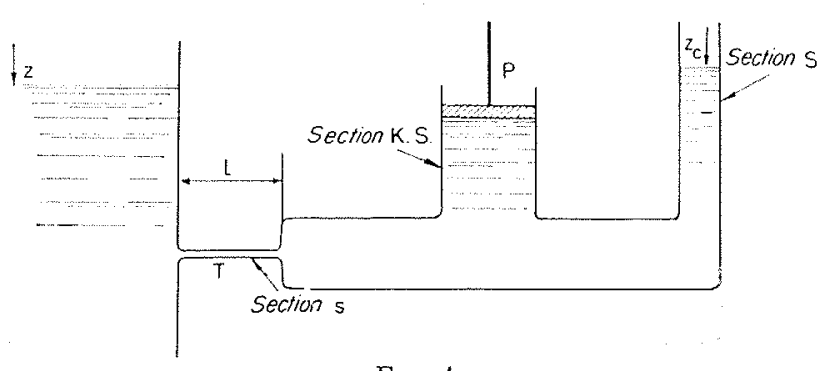

FIG. 4.

et on lui donne un mouvement vertical égal à :

$$
Z(t)-\frac{K-1}{\mathrm{~K}} z(t) .
$$

Si K est assez grand par rapport à 1, l'inertie de l'eau et la perte de charge visqueuse dans le tuyau ' $\mathrm{T}$ sont telles que l'on a la relation (6), avec :

$$
\mathrm{R}=\frac{l}{g} \frac{8 \pi \nu}{s} \frac{\mathrm{KS}}{s} \quad \mathrm{~L}=\frac{l}{g} \frac{\mathrm{K} \mathrm{S}}{s}
$$

Ce système a l'inconvénient, par rapport au dispositif électrique, de n'être pas réglable aussi facilement.

\section{CONCLUSION}

L'appareillage décrit ci-dessus a été expérimenté dans toutes ses parties et son fonctionnement s'est révélé satisfaisant. Sa forme définitive n'a pas encore été fixée, mais il nous a paru intéressant d'en présenter dès maintenant les principes. Nous comptons, dès que nous le pourrons donner une description détaillée de l'appareil définitif et des résultats qu'il aura permis d'obtenir.

\section{I S C U S S I ON}

M. Valembors précise, sur la demande de M. le Président, que l'appareil décrit dans sa communication n’a pas encore fonctionné dans son ensemble, mais que la plus grande partie de ses éléments ont été construits et essayés.

M. le Président souligne l'intérêt de la comparaison de cet appareil avec ceux qui ont été utilisés jusqu'ici, compte tenu des dimensions du modèle réduit à marée qu'il s'agit d'alimenter.

M. le Président remercie M. Vatenrors de sa très intéI'essante communication. 